\title{
Acoustic Radiation Force Impulse Elastography: A Non-Invasive Alternative to Liver Biopsy
}

\author{
Hirokazu Takahashi ${ }^{1}$ et al. ${ }^{*}$ \\ ${ }^{1}$ Department of Internal Medicine, Saga Medical School, Nabeshima, Saga, \\ Japan
}

\section{Introduction}

The prognosis of chronic liver disease depends on the progression of liver fibrosis. Liver biopsy is recommended as the gold standard method for determining fibrosis stage, prognosis and therapeutic indications in patients with chronic liver disease. However, liver biopsy is an invasive procedure associated with a risk of potentially serious complications. Approximately 1-3\% of patients require hospitalization for complications, and a quarter of them report pain after percutaneous liver biopsy. The diagnostic accuracy of liver biopsy for assessing liver fibrosis is influenced by the quality of the biopsy samples. In addition, there are several absolute or relative contraindications to liver biopsy, including severe coagulopathy. A safe and non-invasive alternative to liver biopsy is therefore needed for assessing liver fibrosis in daily clinical practice.

\section{Principle of acoustic radiation force impulse}

Acoustic radiation force impulse (ARFI) imaging is a radiation force-based imaging method that is provided by conventional B-mode ultrasonography (Acuson S2000; SIEMENS Medical Solutions) (Fig. 1). ARFI imaging involves transmission of an initial ultrasonic pulse at diagnostic intensity levels to obtain a baseline signal for later comparison. A shortduration (approximately $0.3 \mathrm{~s}$ ), high-intensity acoustic 'pushing pulse' is then transmitted by the same transducer, followed by a series of diagnostic intensity pulses, which are used to track the displacement of the tissue caused by the pushing pulse (Nightingale et al., 2001, Palmeri et al., 2005, Dahl et al., 2007). The response of the tissue to the radiation force is observed using conventional B-mode imaging pulses, and it is possible to display the quantitative shear-wave velocity (Vs; m/s) of ARFI displacement. This velocity $(\mathrm{m} / \mathrm{s})$ is proportional to the square root of tissue elasticity. Because the velocity of the shear wave depends on tissue stiffness, it is possible to apply ARFI technology to elastography. This

\footnotetext{
${ }^{*}$ Naofumi Ono ${ }^{2}$, Yuichiro Eguchi ${ }^{1}$, Toshihiko Mizuta ${ }^{1}$, Keizo Anzai $^{1}$, Atsushi Miyoshi ${ }^{3}$, Masato Yoneda ${ }^{4}$, Atsushi Nakajima ${ }^{4}$ and Kazuma Fujimoto ${ }^{1}$.

1 Department of Internal Medicine, Saga Medical School, Nabeshima, Saga, Japan,

2 Department of Clinical Gastroenterology, Eguchi Hospital, Mikazuki, Ogi, Saga, Japan,

3 Department of Surgery, Saga Medical School, Nabeshima, Saga, Japan,

4 Division of Gastroenterology, Yokohama City University Hospital, Yokohama, Japan.
} 
technology was named "Virtual Touch Tissue Quantification" by SIEMENS. Previous reports have also referred to it as ARFI elastography or ARFI elastometry. In this chapter, we use the term ARFI elastography.

\section{Protocol of ARFI elastography}

ARFI imaging involves targeting an anatomic region to be examined for its elastic properties using a region-of-interest (ROI) cursor measuring $10 \times 5 \mathrm{~mm}$ at the same time as or after performing real-time B-mode imaging (Fig. 2). This enables a comprehensive range of tissue strain analytic parameters to be assessed, allowing quantitative values of the mechanical stiffness (elasticity) properties of tissues to be measured. In general, the stiffer a region of tissue, the greater the shear-wave speed as it travels through this region. ARFI elastography is performed using a curved array at $4 \mathrm{MHz}$ for B-mode imaging. The right lobe of the liver is examined through the intercostal space with the patient lying in the dorsal decubitus position, with their right arm maximally abducted. A measurement depth of 2-3 cm below the liver capsule is chosen to standardize the examination. No consensus has yet been reached regarding the ROI position and the approach point on the body surface, but our experience indicates that measurement at 2-3 cm depth from the right intercostals seems to produce stable results. According to a recent report, there was no significant difference in ARFI measurements between the intercostal and abdominal approaches; however, the intercostal approach had the highest success rates (Popescu et al., 2011). Although different values of ARFI measurements were reported for each depth under the liver capsule, the differences were not significant (Sporea et al., 2010). Boursier et al. reported that the reproducibility of measurements in the right lobe was high, but measurements in the left lobe included cases with outliers (Boursier et al., 2010). Further studies are needed to determine the depth of ROI for the appropriate stratification of liver fibrosis stages. The Acuson S2000 has a memory capability, and more than five successful acquisitions should thus be performed for each patient; the median or mean value can then be calculated to produce a reliable measurement.

\section{Reproducibility of ARFI elastography}

According to previous reports, ARFI elastography has high reproducibility. Boursier et al. reported that the interobserver reproducibility of ARFI was excellent (interclass correlation coefficient $=0.91$ ) and no training effect was observed in terms of ARFI measurements (Boursier et al., 2010). Friedrich-Rust et al. found 87\% agreement regarding ARFI elastography-derived stages between two examiners (Friedrich-Rust et al., 2009).

\section{ARFI elastography in normal healthy subjects}

Several studies have determined the normal range of ARFI measurements for healthy subjects. Popescu et al. reported a mean shear-wave velocity of $1.15 \pm 0.21 \mathrm{~m} / \mathrm{s}$ (Popescu et al., 2011). The study by Takahashi et al. included 25 healthy controls, and found a mean shear-wave value of $1.08 \pm 0.13 \mathrm{~m} / \mathrm{s}$ (Takahashi et al., 2010). The mean shear-wave velocity in the study by Horster et al., which included 68 healthy volunteers with a mean age of 28 years, was $1.19 \mathrm{~m} / \mathrm{s}$ (range, 0.77-1.63 m/s) (Horster et al., 2010), while Goertz et al. obtained a mean value of $1.09 \mathrm{~m} / \mathrm{s}$ (range, $0.79-1.32 \mathrm{~m} / \mathrm{s}$ ) in 20 healthy subjects. Kim et al. included 133 subjects with normal livers in their series and derived a mean value of $1.08 \pm 0.15 \mathrm{~m} / \mathrm{s}$ (Goertz et al., 2010, Kim et al., 2010). No correlations between ARFI values and age, gender or body mass index (BMI) have been identified. 


\section{Diagnostic accuracy for liver fibrosis}

Ultrasonography-based evaluation offers several advantages, including ready availability, real-time examination, relatively low cost and lack of ionizing radiation. Recent studies have indicated the clinical feasibility of ARFI elastography for assessing liver fibrosis (Table 1). The results demonstrated that shear-wave velocity measured by ARFI elastography offers a reliable and non-invasive method for estimating liver fibrosis in chronic liver diseases. In particular, liver stiffness assessed by shear-wave velocity was significantly correlated with liver fibrosis according to the pathological scoring system. Furthermore, the staging of liver fibrosis in chronic liver diseases could be accurately classified based on shear-wave velocity. Friedrich-Rust et al. reported that the area under the receiver operating characteristic curve for the accuracy of ARFI elastography was 0.91 for the diagnosis of both moderate fibrosis $(\mathrm{F} \geq 2)$ and cirrhosis, respectively. According to their report, the cut-off value for liver cirrhosis was $1.75 \mathrm{~m} / \mathrm{s}$ in all subjects, and $1.75 \mathrm{~m} / \mathrm{s}$ in subjects with hepatitis C virus (HCV) infection (Friedrich-Rust et al., 2009). Other reports have identified variable cut-off values for cirrhosis, ranging from 1.6-1.95 m/s (Table 1). In the studies by Takahashi et al. and Sporea et al., which included $47(85 \%)$ and 54 (76\%) HCV-positive subjects, respectively, the cut-off value for cirrhosis was $1.8 \mathrm{~m} / \mathrm{s}$ (Takahashi et al., 2010, Sporea et al., 2010). The study by Yoneda et al. included subjects with non-alcoholic fatty liver disease (NAFLD), and found a cut-off value for cirrhosis of $1.9 \mathrm{~m} / \mathrm{s}$, which was higher than in other studies (Yoneda et al., 2010). It is possible that differences in background chronic liver diseases might account for the variations in cut-off values. In addition, different distributions of fibrosis stages among subjects in different studies may affect the results of ROC analysis, and direct comparison of cut-off values among studies should therefore be avoided. The Obuchowski measure, which allows for differential penalization of more serious misdiagnoses, was recently reported (Obuchowski et al., 2005). This method allows the diagnostic accuracy of ARFI elastography among studies with different distributions of fibrosis stages to be compared (Lambert et al., 2008). However, because the Obuchowski measure is statistically technical, analysis with commercially-based medical statistical software might be difficult. Further studies on the correlations between different liver disease etiologies and the results of ARFI elastography are needed, as are standardized measurement protocols and statistical methods. Nonetheless, ARFI elastography appears to offer good diagnostic accuracy for staging liver fibrosis.

\begin{tabular}{|c|c|c|c|c|c|}
\hline & & \multicolumn{3}{|c|}{ AUROC } & $\begin{array}{c}\text { Cut-off for } \\
\text { cirrhosis }\end{array}$ \\
\hline Author & Subjects & FS 0-1vs FS 2-4 & FS 0-2 vs. FS 3-4 & FS 0-3 vs. FS 4 & (m/s) \\
\hline $\begin{array}{c}\text { Friedrich- } \\
\text { Rust et al. }\end{array}$ & HCV, HBV & 0.84 & 0.91 & 0.91 & 1.75 \\
\hline Takahashi et al. & HCV, HBV, NASH & 0.94 & 0.94 & 0.96 & 1.8 \\
\hline Yoneda et al. & NAFLD & - & 0.97 & 0.98 & 1.9 \\
\hline Sporea et al. & HCV, HBV & 0.65 & & 0.87 & 1.8 \\
\hline Goertz et al. & HCV, HBV & 0.85 & 0.92 & 0.87 & - \\
\hline $\begin{array}{c}\text { Fierbinteanu- } \\
\text { Braticevici et al. }\end{array}$ & HCV & 0.92 & 0.99 & 0.99 & 1.95 \\
\hline Rifai et al. & HCV, HBV, NAFLD & - & - & 0.82 & 1.6 \\
\hline
\end{tabular}

Table 1. Diagnostic accuracy of ARFI for liver fibrosis 
AUROC, area under the receiver operating characteristic curve; FS, fibrosis stage; $\mathrm{HCV}$, hepatitis C virus; HBV, hepatitis B virus; NAFLD, non-alcoholic fatty liver disease. Fibrosis stage was assessed using the Metavir scoring system, except in the case of Yoneda et al., who used the method of Brunt.

\section{Factors influencing ARFI elastography measurements}

The degree of liver fibrosis is the most significant factor determining shear-wave velocity. However, several other factors can affect shear-wave velocity. Rifai et al. reported that shear-wave velocity was faster in patients with significant liver inflammation compared with those with no significant inflammation (Rifai et al., 2011). They suggested that liver and spleen sizes were positively correlated with the results of ARFI elastography measurements. Takahashi et al. reported that serum aspartate aminotransferase and alanine aminotransferase levels as well as pathological liver inflammation were positively correlated with ARFI elastography measurements (Takahashi et al., 2010). These results suggest that pathological and serological liver inflammation might affect shear-wave velocity. However, it should be noted that significant proportions of patients in those studies had viral hepatitis, and the severity of pathological fibrosis has been shown to correlate with pathological inflammation in viral hepatitis. Further studies including larger sample sizes are needed to evaluate the impact of inflammation on ARFI elastography measurements in patients with the same severities of fibrosis stage.

Pathological liver steatosis is a significant factor affecting ARFI elastography measurements in NAFLD. Yoneda et al. suggested that shear-wave velocity in NAFLD with no fibrosis was slower than in healthy controls. Additionally, NAFLD with mild liver fibrosis was associated with almost normal shear-wave velocities (Yoneda et al., 2010, Palmeri et al., 2011). They suggested that steatosis might make the liver softer because of fat deposition in the liver parenchyma. Palmeri et al. showed that hepatocyte ballooning and inflammation in NAFLD had no significant effects on shear-wave velocity (Palmeri et al., 2011).

\section{Comparison with other non-invasive methods}

Several scoring systems or indexes based on serum biochemical markers have been proposed for the non-invasive prediction of liver fibrosis in chronic liver disease. Additionally, transient elastography (FibroScan) has recently been developed as a noninvasive method for the evaluation of liver fibrosis, by assessing liver stiffness based on a mechanical wave generated by vibration. The diagnostic accuracy of ARFI has been shown to be comparable with that of previously reported non-invasive scoring systems such as AST to Platelet Ratio Index (APRI), Fib-4 and Forn's index (Takahashi et al, 2010). Several studies have reported both ARFI and FibroScan measurements. The results of ARFI elastography were highly correlated with those of FibroScan measurements, though the success rate of ARFI measurements was higher than that for FibroScan. To the best of our knowledge, few reports have compared the diagnostic accuracies of ARFI and FibroScan, based on the gold standard of liver biopsy. Sporea et al. suggested that the accuracy of ARFI elastography was acceptable but lower than that of FibroScan (Sporea et al., 2010), while Friedrich-Rust et al. and Rifai et al. found no significant differences in diagnostic accuracy between the two techniques (Table 2) (Friedrich-Rust et al., 2009, Rifai et al., 2011). It thus remains unclear which form of elastography is more reliable; however, a prospective 
multicenter study and meta-analysis are currently underway. Nonetheless, ARFI elastography appears to be useful because of its fewer limitations and the integration of elastography and conventional B-mode ultrasonography.

\begin{tabular}{|c|c|c|c|c|c|}
\hline & & \multicolumn{2}{|c|}{ FS 0-1 vs. FS 2-4 } & \multicolumn{2}{c|}{ FS 0-3 vs. FS 4 } \\
\hline Author & Subjects & ARFI & FibroScan & ARFI & FibroScan \\
\hline Friedrich-Rust et al. & HCV, HBV & 0.82 & 0.84 & 0.91 & 0.91 \\
\hline Sporea et al. & HCV, HBV & 0.65 & 0.73 & 0.87 & 0.94 \\
\hline Rifai et al. & HCV, HBV, NAFLD & - & - & 0.82 & 0.84 \\
\hline
\end{tabular}

Table 2. Diagnostic accuracy of ARFI and FibroScan for liver fibrosis

AUROC, area under the receiver operating characteristic curve; FS, fibrosis stage; HCV, hepatitis $C$ virus; $\mathrm{HBV}$, hepatitis B virus; NAFLD, non-alcoholic fatty liver disease. Fibrosis stage was assessed using the Metavir scoring system, except in the case of Yoneda et al., who used the method of Brunt.

\section{Limitations and possible contraindications of ARFI elastography}

No significant limitations or contraindication of ARFI elastography for liver assessment have been reported to date. However, the Japan Society of Ultrasonics in Medicine recommends that ARFI elastography should be avoided after contrast-enhanced ultrasonography with some kinds of contrast agents, because of the risk of cavitation. The limitations of transient elastography, such as narrow intercostal spaces, ascites and obesity, do not appear to apply to the use of ARFI elastography, according to our data and the results of previous reports. However, the success rate of ARFI measurements in subjects with a BMI $>40 \mathrm{~kg} / \mathrm{m}^{2}$ was reportedly only $58 \%$ (Palmeri et al., 2011). Further studies are needed to allow more precise evaluation of the limitations of ARFI elastography.

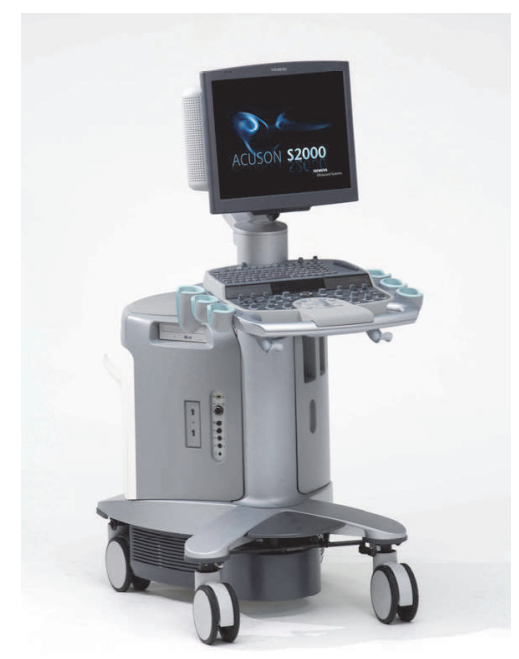

Fig. 1. SIEMENS Acuson S2000 
Acoustic radiation force impulse (ARFI) is a radiation force-based impulse that is provided by conventional B-mode ultrasonography, using an Acuson S2000. The ARFI method can be used for elastography. The technology was named "Virtual Touch Tissue Quantification" by SIEMENS.

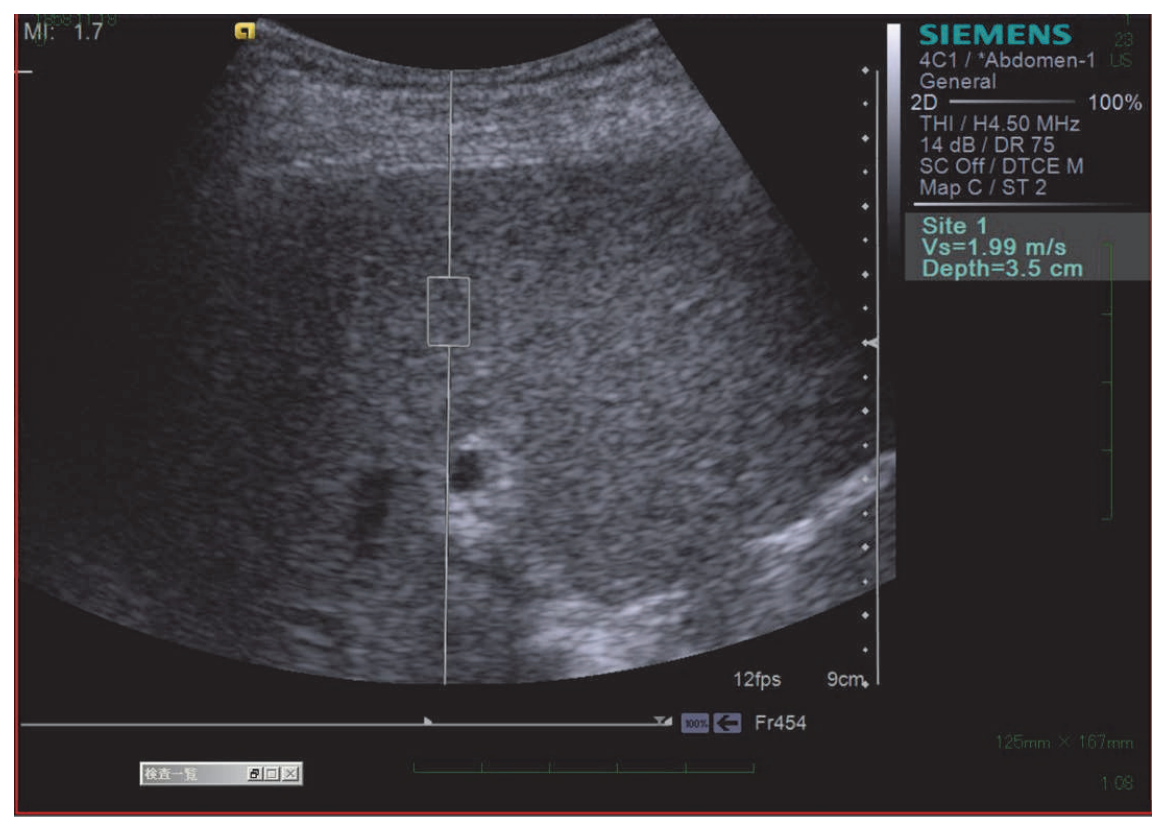

Fig. 2. Measurement window for ARFI elastography

\section{Conclusion}

In conclusion, the novel, non-invasive ultrasonic ARFI elastography technique, coupled with conventional ultrasonography, could offer a reliable method for the assessment of liver fibrosis in patients with chronic liver diseases. ARFI elastography could be useful for realtime evaluation of liver fibrosis as an alternative to liver biopsy.

\section{Acknowledgments}

The authors would like to thank Ms Yukie Watanabe, Ms Chieko Ogawa, all the staff at Saga Medical School Hospital and Yokohama City University Hospital, and Ritsuko Fukusima at Mochida SIEMENS Medical Systems, Japan, for their assistance and excellent advice.

\section{References}

Boursier J, Isselin G, Fouchard-Hubert I, Oberti F, Dib N, Lebigot J, Bertrais S, Gallois Y, Calès P, Aubé C. Eur J Gastroenterol Hepatol. 2010;22:1074-84. Acoustic radiation force impulse: a new ultrasonographic technology for the widespread noninvasive diagnosis of liver fibrosis. 
Dahl JJ, Pinton GF, Palmeri ML, Agrawal V, Nightingale KR, Trahey GE.. A parallel tracking method for acoustic radiation force impulse imaging. IEEE Trans Ultrason Ferroelectr Freq Control. 2007;54:301-12.

Fierbinteanu-Braticevici C, Andronescu D, Usvat R, Cretoiu D, Baicus C, Marinoschi G. Acoustic radiation force imaging sonoelastography for noninvasive staging of liver fibrosis. World J Gastroenterol. 2009;15:5525-32.

Friedrich-Rust M, Wunder K, Kriener S, Sotoudeh F, Richter S, Bojunga J, Herrmann E, Poynard T, Dietrich CF, Vermehren J, Zeuzem S, Sarrazin C. Liver fibrosis in viral hepatitis: noninvasive assessment with acoustic radiation force impulse imaging versus transient elastography.Radiology. 2009;252(2):595-604.

Goertz RS, Zopf Y, Jugl V, Heide R, Janson C, Strobel D, Bernatik T, Haendl T. Measurement of liver elasticity with acoustic radiation force impulse (ARFI) technology: an alternative noninvasive method for staging liver fibrosis in viral hepatitis. Ultraschall Med. 2010;31:151-55.

Horster S, Mandel P, Zachoval R, Clevert DA. Comparing acoustic radiation force impulse imaging to transient elastography to assess liver stiffness in healthy volunteers with and without valsalva manoeuvre. Clin Hemorheol Microcirc. 2010;46:159-68.

Kim JE, Lee JY, Kim YJ, Yoon JH, Kim SH, Lee JM, Han JK, Choi BI. Acoustic radiation force impulse elastography for chronic liver disease: comparison with ultrasound-based scores of experienced radiologists, Child-Pugh scores and liver function tests. Ultrasound Med Biol. 2010;36:1637-43.

Lambert J, Halfon P, Penaranda G, Bedossa P, Cacoub P, Carrat F. How to measure the diagnostic accuracy of noninvasive liver fibrosis indices: the area under the ROC curve revisited. Clin Chem. 2008;54:1372-8.

Nightingale KR, Palmeri ML, Nightingale RW, Trahey GE. On the feasibility of remote palpation using acoustic radiation force. J Acoust Soc Am 2001.110: 625-34.

Obuchowski NA. Estimating and comparing diagnostic tests' accuracy when the gold standard is not binary. Acad Radiol. 2005;12:1198-204.

Palmeri ML, Frinkley KD, Zhai L, Gottfried M, Bentley RC, Ludwig K, Nightingale KR. Acoustic radiation force impulse (ARFI) imaging of the gastrointestinal tract. Ultrason Imaging. 2005;27:75-88.

Palmeri ML, Wang MH, Rouze NC, Abdelmalek MF, Guy CD, Moser B, Diehl AM, Nightingale KR.Noninvasive Evaluation of Hepatic Fibrosis using Acoustic Radiation Force-Based Shear Stiffness in Patients with Nonalcoholic Fatty Liver Disease. J Hepatol. 2011 Jan 20 (Epub ahead of print. URL: http://www.sciencedirect.com/science/article/pii/S0168827811000079).

Popescu A, Sporea I, Sirli R, Bota S, Focşa M, Dănilă M, Nicoliță D, Martie A, Sendroiu M, Juchiş A.The mean values of liver stiffness assessed by Acoustic Radiation Force Impulse elastography in normal subjects. Med Ultrason. 201;13:33-7.

Rifai K, Cornberg J, Mederacke I, Bahr MJ, Wedemeyer H, Malinski P, Bantel H, Boozari B, Potthoff A, Manns MP, Gebel M. Clinical feasibility of liver elastography by acoustic radiation force impulse imaging (ARFI).Dig Liver Dis. 2011;43:491-7.

Sporea I, Sirli R, Popescu A, Danilă M. Acoustic Radiation Force Impulse (ARFI)--a new modality for the evaluation of liver fibrosis. Med Ultrason. 2010;12:26-31.

Takahashi H, Ono N, Eguchi Y, Eguchi T, Kitajima Y, Kawaguchi Y, Nakashita S, Ozaki I, Mizuta T, Toda S, Kudo S, Miyoshi A, Miyazaki K, Fujimoto K. Evaluation of 
acoustic radiation force impulse elastography for fibrosis staging of chronic liver disease: a pilot study. Liver Int. 2010;30:538-45.

Yoneda M, Suzuki K, Kato S, Fujita K, Nozaki Y, Hosono K, Saito S, Nakajima A. Nonalcoholic fatty liver disease: US-based acoustic radiation force impulse elastography. Radiology. 2010;256:640-7. 


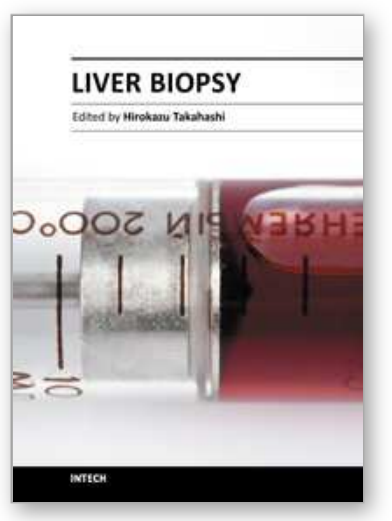

\author{
Liver Biopsy \\ Edited by Dr Hirokazu Takahashi
}

ISBN 978-953-307-644-7

Hard cover, 404 pages

Publisher InTech

Published online 06, September, 2011

Published in print edition September, 2011

Liver biopsy is recommended as the gold standard method to determine diagnosis, fibrosis staging, prognosis and therapeutic indications in patients with chronic liver disease. However, liver biopsy is an invasive procedure with a risk of complications which can be serious. This book provides the management of the complications in liver biopsy. Additionally, this book provides also the references for the new technology of liver biopsy including the non-invasive elastography, imaging methods and blood panels which could be the alternatives to liver biopsy. The non-invasive methods, especially the elastography, which is the new procedure in hot topics, which were frequently reported in these years. In this book, the professionals of elastography show the mechanism, availability and how to use this technology in a clinical field of elastography. The comprehension of elastography could be a great help for better dealing and for understanding of liver biopsy.

\title{
How to reference
}

In order to correctly reference this scholarly work, feel free to copy and paste the following:

Hirokazu Takahashi, Naofumi Ono, Yuichiro Eguchi, Toshihiko Mizuta, Keizo Anzai, Atsushi Miyoshi, Masato Yoneda, Atsushi Nakajima and Kazuma Fujimoto (2011). Acoustic Radiation Force Impulse Elastography: A Non-Invasive Alternative to Liver Biopsy, Liver Biopsy, Dr Hirokazu Takahashi (Ed.), ISBN: 978-953-307-644-7, InTech, Available from: http://www.intechopen.com/books/liver-biopsy/acoustic-radiation-force-impulseelastography-a-non-invasive-alternative-to-liver-biopsy

\section{INTECH}

open science | open minds

\section{InTech Europe}

University Campus STeP Ri

Slavka Krautzeka 83/A

51000 Rijeka, Croatia

Phone: +385 (51) 770447

Fax: +385 (51) 686166

www.intechopen.com

\section{InTech China}

Unit 405, Office Block, Hotel Equatorial Shanghai

No.65, Yan An Road (West), Shanghai, 200040, China

中国上海市延安西路65号上海国际贵都大饭店办公楼405单元

Phone: +86-21-62489820

Fax: $+86-21-62489821$ 
(C) 2011 The Author(s). Licensee IntechOpen. This chapter is distributed under the terms of the Creative Commons Attribution-NonCommercialShareAlike-3.0 License, which permits use, distribution and reproduction for non-commercial purposes, provided the original is properly cited and derivative works building on this content are distributed under the same license. 